\title{
Displasia cemento óssea florida
}

\author{
Danilo Barral Araújo ${ }^{1}$ \\ Isaac Vieira Queiroz ${ }^{1}$ \\ Pedro Romero ${ }^{2}$ \\ Antonio Fernando Pereira Falcão ${ }^{3}$
}

\begin{abstract}
Resumo
A Displasia Cemento Óssea Florida (DCOF) é uma lesão controvertida em relação ao seu diagnostico, pelos seus aspectos clínicos e radiográficos muito semelhantes a outras lesões ósseas esclerosantes. Acomete principalmente a mandíbula de mulheres negras com idade acima de quarenta anos. Apresenta um aspecto radiográfico de massas densamente radiopacas, entremeadas por faixas radiolúcidas, sem limite definido. Pode desenvolver expansão óssea alveolar, supuração e fistulação, caso esteja associada à infecção secundaria (osteomielite), que ocorre devido à exposição da massa óssea avascular à cavidade bucal.
\end{abstract}

Palavras-chave: lesões escleróticas; escleroses ósseas; cemento.

\section{INTRODUÇÃO}

Este trabalho objetiva relatar uma revisão de literatura sobre a Displasia Cemento Óssea Florida, uma lesão com controvérsia em relação ao seu diagnóstico, já que seus aspectos clínicos e radiográficos são muito semelhantes a outras lesões, como, por exemplo, Osteomielite Crônica Esclerosante difusa, Cementoma Giganteforme familiar e doença de Paget. Assim, para sua elucidação, ilustrar-se-á com um caso clínico.

É uma lesão, que acomete principalmente a mandíbula de mulheres negras, de meia idade. Apresenta o aspecto radiográfico de áreas densamente radiopacas, rodeadas por faixa radiolúcida sem limite definido. Desenvolve uma expansão óssea alveolar e drenagem com processo fistuloso.

Uma infecção secundaria (osteomielite) ocorre caso haja a exposição da massa óssea alveolar à cavidade bucal devido a uma extração dentaria, uso de prótese mal adaptada e biopsias.

É importante o estudo dessa patologia para diminuir a controversa a seu respeito, favorecendo um diagnostico cada vez mais preciso.

\section{REVISÃO DE LITERATURA}

A displasia cemento óssea florida (DCOF) foi descrita pela primeira vez por Melrose e cola-

\footnotetext{
${ }^{1}$ Academico do Curso de Odontologia da Universidade Federal da Bahia. Salvador - BA

2 Preceptor de Estágio Supervisionado. Serviço de Urgência. Faculdade de Odontologia. Universidade Federal da Bahia. Salvador - BA

${ }^{3}$ Professor Doutor da disciplina Estomatologia. Universidade Federal da Bahia. Salvador - BA

Correspondência para / Correspondence to:

Antonio Fernando Pereira Falcão

Faculdade de Odontologia

Universidade Federal da Bahia

Rua Araújo Pinho s/n - Canela

40.000-000 - Salvador Bahia Brasil

E-mail: afpfalcao@hotmail.com
} 
boradores em 1976. Constatou-se sua maior incidência em mulheres negras de meia idade, fato confirmado por diversos estudos (MELROSE; ABRAMS; MILLS, 1976; ARIJI et al., 1994).

A DCOF é uma condição não neoplásica, assintomática, benigna, caracterizada por múltiplas áreas escleróticas simetricamente distribuídas nos maxilares, com marcante tendência ao envolvimento bilateral. $\mathrm{Na}$ grande maioria das vezes, envolve regióes contíguas aos dentes e se restringe ao osso alveolar, não sendo incomum encontrar um envolvimento extenso das regiōes posteriores dos quatro quadrantes. A doença pode ser completamente assintomática e, em tais casos, é descoberta somente quando radiografias são realizadas com outros fins, como também o paciente pode relatar dor de baixa intensidade e uma fístula na mucosa alveolar. Uma ou mais áreas dos maxilares podem exibir algum grau de expansão óssea. Tanto as áreas dentadas como edêntulas podem ser afetadas, e o envolvimento parece não estar relacionado à presença ou ausência de dentes (SOARES et al., 1998; NEVILLE et al., 1998; BEYLOUNI et al., 1998).

Por causa da apresentação de sintomas usualmente associados à exposição de massas escleróticas na cavidade bucal, devem ser evitadas biopsia e extração dentaria (BEYLOUNI et al., 1998).

A patogênese dessa complicação da DCOF poderia ser relacionada à escassa irrigação sanguínea no osso afetado e às massas escleróticas avasculares, particularmente susceptíveis à infecção secundária (MELROSE; ABRAMS; MILLS, 1976; SCHNEIDER; MESA; BRICMAN, 1987).

É uma displasia limitada aos ossos gnáticos. Não há outra evidencia dessa doença, seja laboratorial, seja radiográfica em nenhuma outra parte do corpo humano (NEVILLE et al., 1998).

Enquadra-se no conjunto das lesões fibroósseas. Nos últimos anos, essa lesão tem atraído um crescente interesse dos patologistas, com o objetivo de distinguir definitivamente essa patologia e de pôr fim às discussões diagnósticas decorrentes da superposição de suas características (SOARES et al., 1998).
A DCOF é uma condição incomum, prevalente em mulheres negras, de causa desconhecida (WALDRON, 1993; NEVILLE et al., 1998). É constituída por uma proliferação fibroblástica associada a trabéculas irregulares de osso e material irregular semelhante a cemento (WALDRON, 1993).

$\mathrm{O}$ termo lesão fibro-óssea não indica um diagnóstico específico, apenas representa um grupo extenso de várias entidades. Embora não exista uma classificação completamente satisfatória, é geralmente aceito que as lesões fibro-ósseas benignas da região bucomaxilofacial podem ser divididas em três categorias: displasia fibrosa, neoplasmas fibro-ósseos benignos e lesões reativas (SOARES et al., 1998).

Segundo Soares e colaboradores (1998), a DCOF é, na realidade, uma forma exuberante da displasia cemento óssea periapical, lesão relativamente comum, que ocorre nos ápices dos dentes com vitalidade. Mulheres negras de meia idade são afetadas com mais frequiência, e a maior parte dos casos apresenta lesōes múltiplas que envolvem o ápice dos dentes ântero-inferiores e raramente atinge a maxila. As lesões aparecem no ligamento periodontal ou próximas a ele e, não comprometendo a fixação dentaria, não provocam mobilidade. Por vezes, as lesões localizadas junto ao forame mentoniano parecem comprimir o nervo homônimo, produzindo dor, parestesia e ate anestesia. Pode apresentar padrão radiográfico variado, pois, à medida que a lesão vai cronificando, mais calcificada se torna.

A ocorrência dessa lesão está associada ao ápice dentário em continuidade com o ligamento periodontal ou em regióes de extração dentaria. (SOARES et al., 1998)

Apesar de a OMS, desde 1992, considerar o cementoma giganteforme familiar (CGF) e a DCOF sinônimos e de os achados histológicos serem semelhantes, os estudos demonstram diferenças marcantes entre as duas entidades, pois o CGF apresenta caráter autossômico dominante, ocorrendo exclusivamente em brancos, na primeira ou segunda década de vida, com crescimento relativamente rápido, resultando em deformidade facial proeminente (NEVILLE et al., 1998).

No diagnostico diferencial da DCOF, deve-se incluir doença de Paget e osteomielite 
crônica esclerosante difusa (OCED). A doença de Paget pode acometer vários ossos e, de modo geral, apresenta alterações sanguíneas (elevação da fosfatase alcalina em estagio ativo), enquanto as lesões da DCOF são restritas às áreas dentadas dos maxilares e não apresentam significantes anormalidades bioquímicas (MELROSE; ABRAMS; MILLS, 1976; SCHNEIDER; MESA; BRICMAN,1987; ARIJI et al., 1994).

É importante termos em mente que o diagnóstico da DCOF é baseado principalmente nos achados clínicos e radiográficos, não havendo necessidade, em grande parte dos casos de biópsia para sua confirmação. $\mathrm{Na}$ medida do possível, devemos evitá-la, pois procedimentos invasivos nas áreas envolvidas, tais como extração dentaria, biópsia óssea e tratamento endodôntico sem antibióticoterapia previa têm sido associados com ocorrência de osteomielite, como uma complicação do quadro clínico (ARIJI et al., 1994; NEVILLE et al., 1998; BEYLOUNI et al., 1998; QUIRINO, 2000).

Radiograficamente, apresenta-se como áreas radiopacas irregulares, lobulares e pouco demarcadas. (SOARES et al., 1998)

Quando os pacientes estão livres de sintomas, as radiografias convencionais mostram massas radiopacas difusas em múltiplos quadrantes, por todo processo alveolar (ARIJI et al., 1994). A distribuição lobular das irregularidades radiopacas, através do processo alveolar, com pobre definição de zonas, demonstra aspecto de "chão de vidro" (MELROSE; ABRAMS; MILLS, 1976; BEYLOUNI et al., 1998).

O tecido lesionado consiste em uma massa densa esclerótica, que tem sido interpretada como sendo cemento e osso. A maioria das lesões típicas é altamente radiopaca e lobular na configuração. Essas áreas lobulares densas são freqüentemente entremeadas com alterações mistas radiotransparentes e radiopacas menos definidas. Com o tempo, essas áreas tendem a ficar cada vez mais calcificadas (MELROSE; ABRAMS; MILLS, 1976; NEVILLE et al., 1998; WALDRON, 1993; BEYLOUNI et al., 1998).
As áreas radiotransparentes mais nitidamente definidas, na exploração cirúrgica, provam ser cistos ósseos simples. Os cistos podem ser solitários, como podem representar posiçōes relativamente grandes dentro da lesão. Esses cistos freqüentemente não se curam tão rapidamente quanto cistos em pacientes mais jovens, que não apresentam DCOF (NEVILLE et al., 1998).

A tomografia computadorizada (TC), pela sua capacidade de proporcionar perspectivas axiais, frontais e sagitais, tem seu conhecimento largamente aproveitado na evolução dessas lesões. A reconstrução tridimensional dessas áreas ósseas tem sido um achado muito importante. Áreas hiperdensas, rodeadas por uma camada hipodensa, estão localizadas ao redor dos ápices dos respectivos dentes (BEYLOUNI et al., 1998).

Muitas lesões têm se diferenciado de DCOF, e a imagem da TC pode ser usada para distingui-la de outras lesões, que podem exibir aparência esclerótica semelhante em radiografias convencionais (BEYLOUNI et al., 1998).

Endostoses, exostoses, massas de alta densidade são facilmente observadas em TC, bem como em radiografias oclusais, e são encontradas em continuidade com o osso cortical. Tumores odontogênicos, especialmente o fibroma cemento ossificante, exibem geralmente maior expansão buco lingual do que o DCOF (BEYLOUNI et al., 1998; ARIJI et al., 1994).

$\mathrm{O}$ valor do HU (Unidades Hounsfield) das massas hiperdensas pode ser benéfico no diagnóstico diferencial, por exemplo. Esse valor em um Odontoma será bem maior que o da DCOF, já que o primeiro apresenta esmalte em sua constituição (ARIJI et al., 1994).

Esse valor, em áreas císticas, pode ser usado para distinguir DCOF de queratocistos odontogênicos. Os queratocistos odontogênicos, algumas vezes, contêm massas de queratina no seu interior, cujo valor do HU é mais alto que o encontrado em áreas císticas da DCOF (ARIJI et al., 1994). Do ponto de vista microscópico é constituída de material densamente calcificado, lembrando cemento secundário, com poucas 
células e lacunas preenchidas por tecido conjuntivo e poucos vasos sanguíneos. A reduzida vascularização faz com que esse osso esclerótico responda mal ao trauma e a bactérias bucais, o que pode ocasionar uma osteomielite (SOARES et al., 1998).

A reabsorção das raízes dos dentes apresentava-se em poucos casos, estes acima de seis anos (MELROSE; ABRAMS; MILLS, 1976).

Histopatologicamente, as lesões mostram proliferação fibroblástica associada a trabéculas irregulares de osso trançado e material semelhante ao cemento. As massas lobulares densamente escleróticas são compostas de glóbulos em camadas, ou fundidas, de material relativamente acelular, interpretado pela maioria dos patologistas como representante do cemento (NEVILLE et al., 1998). Essas leões são consideradas como displasias ósseas, porque o cemento se mantém indistinguível do osso (ARIJI et al., 1994).

Nos pacientes assintomáticos, bem como naqueles pacientes portadores de próteses totais, o melhor tratamento consiste em exames periódicos com profilaxia e reforço das instruçōes de higiene bucal, para controlar a doença periodontal e prevenir a perda dos dentes. Para os pacientes que apresentam alguma sintomatologia, o manejo é mais difícil. Nesse estágio, um componente inflamatório está presente, e o processo é basicamente uma osteomielite crônica, que envolve cemento e osso displásico. Antibióticos podem estar indicados, mas não necessariamente serão efetivos. A sequetração de massas de material tipo cemento esclerótico podem ocorrer lentamente, seguida de cicatrização. A eliminação desses seqüestros pode acelerar a cicatrização (NEVILLE et al., 1998).

A condução da sintomatologia do paciente é mais difícil por causa da inflamação crônica e de infecçôes desenvolvidas dentro do tecido denso mineralizado. Quando antibióticos são administrados, têm um pobre padrão de difusão nos tecidos e têm pouca efetividade. Os seqüestros da massa de cemento ocorrem vagarosamente, seguidos de cura da área (ARIJI et al.,1994).

\section{ILUSTRAÇÃO DE UM CASO CLÍNICO}

A paciente ASS, sexo feminino, cor negra, sessenta e três (63) anos, chegou ao ambulatório da disciplina de Estomatologia I da Faculdade de Odontologia da Universidade Federal da Bahia. Portadora de hipertensão arterial, no momento da anamnese apresentou pressão arterial de 160x100mmHg, pulso de 69bpm e freqüência respiratória de 25ipm. Faz uso do Ancoron. Relata distúrbios cardiovasculares, e informa que os irmãos apresentam os mesmos problemas.

A referida paciente deu entrada ao ambulatório, solicitando realização de extração dos dentes, únicos no arco superior $(2.2 ; 2.3$; $2.4 ; 2.5$ ), sendo que o 2.2 se apresentava como resto radicular, fraturado há aproximadamente dois anos e reabilitação protética, após exodontia desses dentes remanescentes. Desde então, apresenta sintomatologia dolorosa.

Ao exame físico intrabucal, verificou-se o já relatado. É importante ressaltar que a paciente apresentava seqüela cirúrgica na maxila direita, que terminou por promover uma comunicação buco-antro-nasal, devido a hemimaxilectomia por Lesão Central de Células Gigantes. Essa condição provoca incômodos tanto estéticos como funcionais e fonéticos. No arco inferior, foi notada a presença de sete (7) dentes (4.2, $4.3,4.4,4.5,3.2,3.3,3.4)$. Nenhuma das unidades remanescentes apresentava, cárie, restaurações ou mobilidade, e todas se apresentavam com vitalidade pulpar.

Observou-se abaulamento mandibular na região de pré-molares esquerdos até a região de molares direitos; houve também expansão em toda maxila esquerda, por vestibular.

Durante o exame das mucosas, verificouse a presença de fistulação e drenagem purulenta. Devido à infecção, optou-se preliminarmente por um tratamento anti-infeccioso.

Diante dos achados clínicos, foram solicitadas radiografias: panorâmica e periapical. Analisando os resultados radiográficos, detectouse a presença de extensas lesões que acometiam a mandíbula, anteriormente e do lado esquerdo até a região de pré-molar, e do lado direito, estendendo-se ate à região retromolar. Notou- 
se também a presença dessas características na maxila esquerda.

As lesões se apresentavam como densas massas radiopacas lobulares irregularmente delimitadas, separadas do osso adjacente por faixa radiolúcida de limites imprecisos, atingindo múltiplos quadrantes, o que caracterizava, dessa forma, a referida displasia.

A partir da ánalise dos exames laboratoriais, constatou-se uma significativa diminuição nos achados dos neutrófilos segmentados, que apresentavam um valor de $32 \%$ (2592). Por outro lado, havia uma elevação nos valores dos linfócitos típicos 58\%

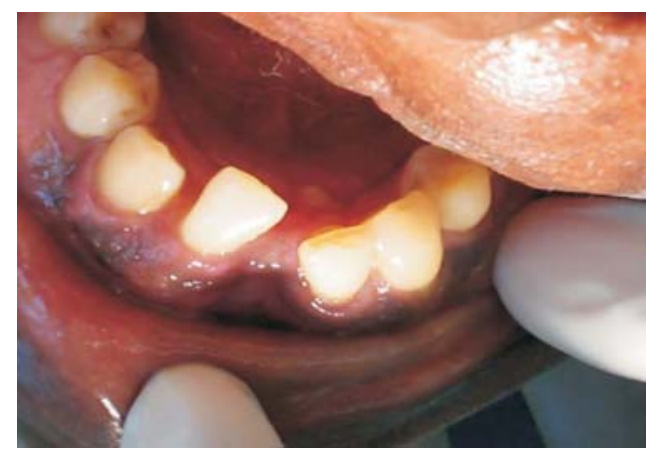

Figura 1 - Arcada dental inferior onde se constata a presença de 7 unidades dentais.

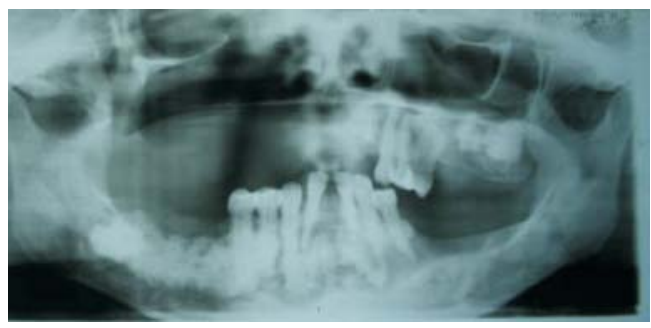

Figura 2 - Radiografia panorâmica na qual são observadas densas massas radiopacas compatíveis com a DCOF e seqüela cirúrgica na maxila direita.

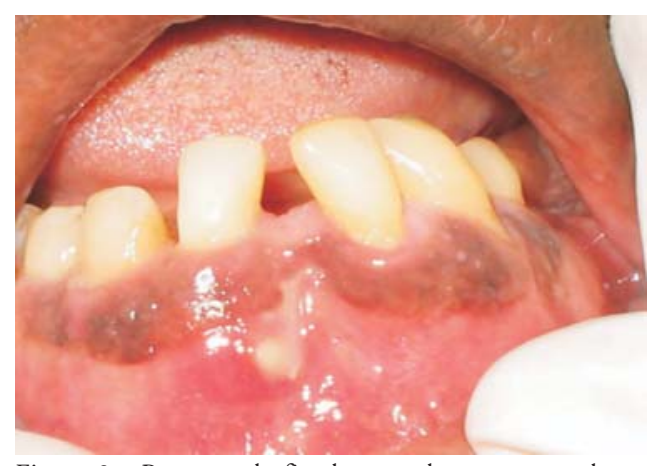

Figura 3 - Presença de fistulação e drenagem purulenta situada na parte anterior da mandíbula.
(4698). Os demais dados de exames laboratoriais, como hemograma, leucograma, índice hemático e ensaios bioquímicos (creatinina, glicemia e uréia), apresentavam-se dentro dos valores normais. A suspeita diagnóstica de Displasia Cemento Óssea Florida foi confirmada pelo exame histopatológico.

$\mathrm{O}$ caso apresentou bom prognóstico. $\mathrm{O}$ plano de tratamento incluiu uma primeira etapa cirúrgica, para uma curetagem óssea e, em seguida, reabilitação implanto-protética.

As figuras 1, 2, 3, 4, 5, 6, 7 e 8 ilustram o caso ora descrito.

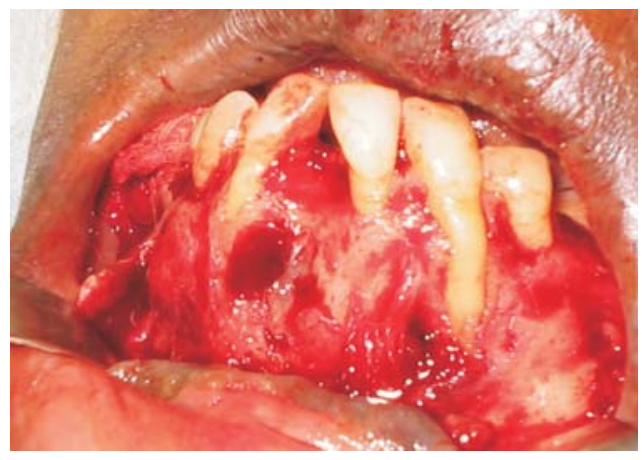

Figura 4 - Presença de fistulação após drenagem purulenta na parte anterior da mandíbula

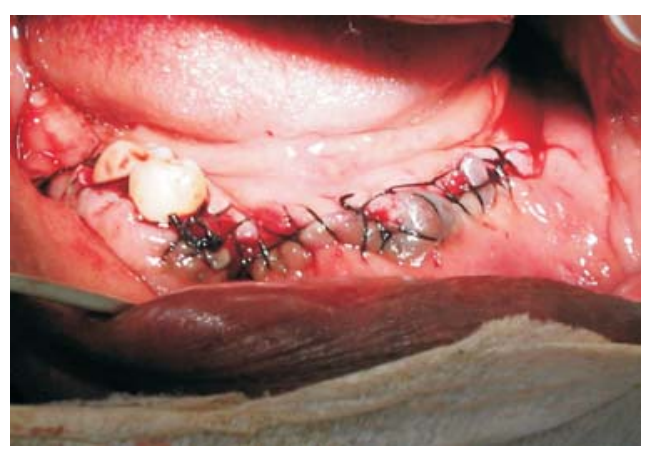

Figura 5 - Primeira etapa cirúrgicas após curetagem óssea e realização das exodontias

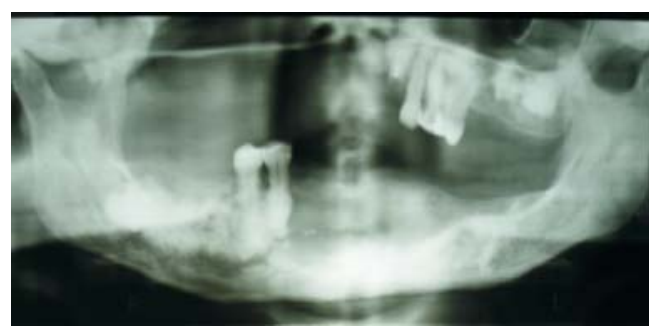

Figura 6 - Radiografia panorâmica que demonstra a conclusão da primeira etapa cirúrgica 


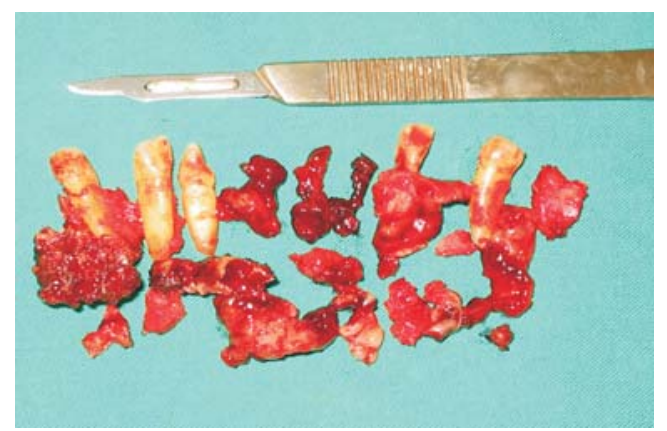

Figura 7 - Resíduos removidos na primeira etapa cirúrgica

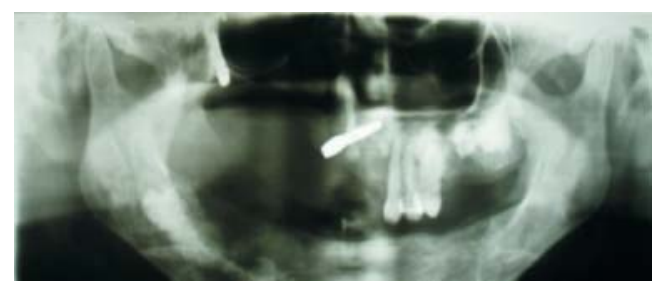

Figura 8 - Radiografia panorâmica da segunda etapa cirúrgica para a reabilitação implanto-protética

\section{DISCUSSÃO}

A DCOF é uma condição incomum, prevalente em mulheres negras, de causa desconhecida, e traz muita discussão a seu respeito (WALDRON, 1993; NEVILLE et al., 1998). Essa situação enquadra-se perfeitamente no caso da paciente ASS, melanoderma e sem história de trauma.

A doença pode ser completamente assintomática e, em tais casos, é descoberta somente quando radiografias são realizadas com outros fins. Uma ou mais áreas dos maxilares podem exibir algum grau de expansão óssea (SOARES et al., 1998). Essas características foram confirmadas durante o acompanhamento do caso, já que a paciente relatou outras queixas sem fazer nenhuma alusão a essa lesão. Notouse moderada expansão óssea na região anterior da mandíbula, apesar de a lesão também aparecer radiograficamente em outros sítios, embora sem expansão nítida.

Tanto as áreas dentadas como as edêntulas podem ser afetadas, e o envolvimento parece não estar relacionado à presença ou ausência de dentes (NEVILLE et al., 1998). Com base análise da radiografia panorâmica, pôde-se constatar a presença da lesão em áreas dentadas e em áreas edêntulas.

Sintomas como dor intensa e drenagem estão quase sempre associados com exposição de massas ósseas escleróticas na cavidade oral (BEYLOUNI et al., 1998). A drenagem foi um aspecto bastante marcante, porém dor e exposição de massas ósseas à cavidade oral não foram relatadas.

A maioria das lesões típicas é altamente radiopaca e lobular na configuração. Essas áreas lobulares densas são freqüentemente entremeadas com alterações mistas radiotransparentes e radiopacas menos bem definidas (NEVILLE et al., 1998). Radiograficamente, a lesão apresentou-se nitidamente lobular radiopaca, entremeada com áreas radiolúcidas menos bem definidas, sem limites precisos.

\section{CONSIDERAÇÕES FINAIS}

A partir dos resultados obtidos, pode-se chegar a uma conclusão de que a DCOF é uma lesão de etiologia desconhecida, que acomete mais freqüentemente mulheres negras de meia idade, sendo comum o acometimento dos maxilares bilateralmente, principalmente na região anterior da mandíbula.

A osteomielite também é uma característica marcante, apesar de ser um sinal secundário, sem necessariamente exposição das massas escleróticas à cavidade oral. Drenagem e supuração também são eventos freqüentes, em conseqüência da osteomielite.

A dor é um sintoma que não deve, brigatoriamente, estar presente.

Não existem relatos de sua presença em nenhum outro sítio do corpo, seja por expressão clínica, radiográfica ou laboratorial.

A lesão apresenta-se com aspecto imaginológico misto: massas radiopacas entremeadas por áreas radiolúcidas.

Deve-se lançar mão de métodos complementares modernos, como, por exemplo, 
a tomografia computadorizada, em associação com os métodos de diagnostico convencionais. Dessa forma, as lesões seriam cada vez mais cedo diagnosticadas, proporcionando menor destrui- ção tecidual e um melhor prognostico para a lesão, o que possibilitaria intervenções cirúrgicas cada vez mais conservadoras.

\title{
Florid cemento osseous dysplasia
}

\begin{abstract}
Florid cemento osseous dysplasia is a controversial disease in terms of diagnosis sinceclinical and radiographic findings are similar to those of other osseous sclerosing lesions. It affects most often the mandible of black women, older than 40 years. The radiographic findings include radiopaque masses and radiolucent strips with badly-defined borders. Alveolar osseous expansion, suppuration and fistulization may develop in case of secondary infection (osteomyelitis), which occurs due to exposure of avascular bone mass to the oral cavity.
\end{abstract}

Keywords: sclerosing lesions; osseous sclerosing lesions; cemento osseous dysplasia.

\section{REFERÊNCIAS}

1. ARIJI, Y. et al. Florid cemento-osseus dysplasia. Radiographic study with special emphasis on computed tomography. Oral Surg oral Med Oral Pathol Radiol Endod., St. Louis, v.78, n.3, p. 391-396, 1994.

2. BEYLUONI, I. et al., Florid cemento osseus dysplasia: report of case documented tomography end 3D imaging. Oral Surg Oral Med Oral Pathol Radiol Endod., v.85, n.6, p.707-711, June. 1998.

3. KRAMER, I. R. H.; PINDBORG, J. J.; SHEAR, M. Neoplasms end other lesions related to bone. In: world Health Organizaition, Histological typing of odontogenic tumors. Berlin: Springer-Verlog, 1992. p. 28-31.

4. MELROSE, R. J.; ABRAMS, A. M.; MILLS, B. H. Florid osseus dysplasia, a clinical pathologic study of thirtyfour cases. Oral Surg Oral Med Oral Pathol Radiol Endod., St. Lois. v.41, p.62-82, 1976.
5. NEVILLE, B. W.; DAMM, D. D.; ALEN, C. M.; BOUQUOT, J. E. Patologia Oral e Maxilofacial. Rio de Janeiro: Guanabara. Koogan. 1998. p.705.

6. QUIRINO, M. R. S. Displasia cemento óssea florida: relato de um caso clínico. Rev. De Biociência da universidade de Taubaté, v.6, n.1, Jan. 2000.

7. SCHNEIDER, L. C.; MESA, M. L.; BRICMAN, J. $\mathrm{H}$. Complications of Endodontic Terapy in florid osseus dysplasia. Oral Surg Oral Med Oral Pathol Radiol Endod., St. Louis, v.64, p.114-16, July. 1987.

8. SOARES, E. C. S. et al., Lesões fibro ósseas: displasia cemento óssea periapical versus Displasia cemento óssea florida. Rev. Fac. Odondol. Porto Alegre, v. 39, n.2, p. 26-30, Dez. 1998.

9. WALDRON, C. A. Fibro osseus lesions of the jaws. J. Oral Maxilofac. Surg, v.5, n.8, p.828-35, Aug. 1993. 